\title{
Stability of the Riemann solution for a $2 \times 2$ strictly hyperbolic system of conservation laws
}

\author{
ANUPAM SEN $^{1}$, T RAJA SEKHAR ${ }^{1, *}$ and DIA ZEIDAN ${ }^{2}$ \\ ${ }^{1}$ Department of Mathematics, Indian Institute of Technology Kharagpur, Kharagpur, India \\ ${ }^{2}$ School of Basic Sciences and Humanities, German Jordanian University, Amman, Jordan \\ e-mail: sen.anupam123@gmail.com; trajasekhar@maths.iitkgp.ac.in; dia.zeidan@gju.edu.jo
}

MS received 29 April 2019; revised 2 September 2019; accepted 6 September 2019

\begin{abstract}
In this work, we study the system of conservation laws that is strictly hyperbolic and whose Riemann solution contains delta shock waves as well as classical elementary waves. In order to study stability, we consider the linear approximation of flux functions with three parameters. The approximation does not affect the structure of Riemann solution. Furthermore, we prove that the solution of the Riemann problem for the approximated system converges to the solution of the original system when the perturbation parameter tends to zero.
\end{abstract}

Keywords. Riemann problem; delta shock wave; strictly hyperbolic system; flux approximation.

\section{Introduction}

We consider the Riemann problem for the following system that arises in nonlinear elasticity and gas dynamics [1]:

$$
\left\{\begin{array}{l}
u_{t}+\left(\frac{u^{2}}{2}\right)_{x}=0, \\
\rho_{t}+((u-1) \rho)_{x}=0, t \geq 0, x \in \mathbb{R},
\end{array}\right.
$$

where $x$ and $t$ represent space and time coordinates, respectively, and $u$ is the background flow field, which carries certain dust particles with density $\rho$. The corresponding solution of the Riemann problem for the system (1) cannot be solved for all the initial data with classical elementary waves. These types of systems occur in gas dynamics and nonlinear elasticity, and have been studied by various authors [1-3]. We refer to [1, 2, 4] for showing how system (1) can be derived as an approximation to the equations of magnetohydrodynamics. There are some nonclassical situations where in contrast to Lax's [5] and Glimm's [6] results, the Cauchy problem for a system of conservation laws fails to contain a weak $L^{\infty}$-solution except for some particular initial data. For this, when we solve the Cauchy problem in this nonclassical situation, it is necessary to introduce a new singular solution called delta shock wave. From (1) we note that the first equation represents the inviscid Burgers equation, whose Riemann solution is the classical entropy solution. If the characteristic velocity $u$ is discontinuous, then the Dirac function is introduced as a part for $\rho$. Nedeljkov and

*For correspondence

Published online: 01 November 2019
Oberguggenberger [1] discussed the interaction of delta shock waves for the system (1).

There are various methods for solving the problem of delta shock waves, like vanishing viscosity method, vanishing pressure limit method, weak asymptotic method, flux approximation method, etc. To construct a delta shock wave solution using vanishing viscosity method we refer to [7-9]. Formation of delta shocks for isentropic and nonisentropic fluids to the Euler equations when pressure vanishes has been studied by Chen and Liu $[10,11]$. The weak asymptotic method for delta shock wave was used in [12-14]. Yin and Sheng [15] discussed delta shock wave solution to relativistic Euler equations for polytropic gases. Mitrovic and Nedeljkov [16] proved that delta shock waves are a limit of shock waves. Physically, a reasonable perturbation can be used to govern some dynamical behaviours of fluids; hence the flux perturbation problem, which plays an important role in the theory, computation and applications, is worth studying. Using flux approximation, Sun [17] studied the limits of Riemann solutions of simplified pressureless Euler system. The flux function approximation for a system of pressureless gas dynamics with two parameters has been discussed by Shen [18]. Sun [19] proved the stability of Riemann solutions for a non-strictly hyperbolic system using flux approximations. Shen and Sun [20] studied singular limits of Riemann solutions to a chemotaxis model using flux perturbation. To control some dynamical behaviour of fluids in a numerical calculation [21] and analytical study [22], the method of flux perturbation is used, which indicates that in the original model some small shear forces are usually ignored. 
In this paper, we consider linear approximation of flux functions in (1) as follows:

$$
\left\{\begin{array}{l}
u_{t}+\left(\frac{u^{2}}{2}+\eta \alpha u\right)_{x}=0, \\
\rho_{t}+((u-1) \rho+\eta \beta u+\eta \gamma \rho)_{x}=0, t \geq 0, x \in \mathbb{R},
\end{array}\right.
$$

where $\alpha, \beta$ and $\gamma$ are arbitrary positive real numbers such that $\alpha>\gamma$ and $\eta>0$ is sufficiently small.

We consider the initial data as

$$
(u, \rho)(x, 0)=\left\{\begin{array}{c}
\left(u_{l}, \rho_{l}\right), x<0 \\
\left(u_{r}, \rho_{r}\right), x>0
\end{array}\right.
$$

where $u_{l, r}$ and $\rho_{l, r}>0$ are given constants.

The system (1) is a particular example of strictly hyperbolic system. It can be seen that (2) is also a strictly hyperbolic system of conservation laws under triangular linear approximation of flux functions. We show that the delta shock wave also appears in the solution of Riemann problem (2) and (3) for some special initial data. Furthermore, we prove rigorously that the limit of the Riemann solution to the problem (2) and (3) converges to the Riemann solution of (1) and (3), when the perturbation parameter $\eta \rightarrow 0$.

We organize this paper in the following way. In section 2, we describe some preliminaries for system (1) and (3) and construct Riemann solution of (1) and (3) with different possible initial data. In section 3, we consider the perturbed Riemann problem (2) with Riemann initial data (3) and Riemann solutions are formed for all possible cases. In section 4, limiting behaviour of Riemann solution to the approximated system (2) is discussed when the perturbation parameter $\eta \rightarrow 0$ and it is proved that it is exactly the same with the corresponding Riemann solution to (1). Finally, conclusions are drawn in section 5 .

\section{Riemann problem for (1) and (3)}

Here we discuss some basic results related to Riemann solution of (1). The characteristic values of (1) are $\lambda_{1}=$ $u-1$ and $\lambda_{2}=u$ and the corresponding right eigenvectors are $\overrightarrow{r_{1}}=(0,1)^{t}$ and $\overrightarrow{r_{2}}=(1, \rho)^{t}$, respectively. Since $\nabla \lambda_{1}$. $\overrightarrow{r_{1}}=0$ and $\nabla \lambda_{2} \cdot \overrightarrow{r_{2}} \neq 0$, the first and second characteristics fields are linearly degenerate and genuinely nonlinear, respectively; $z_{1}=u$ and $z_{2}=\rho e^{-u}$ are the Riemann invariants, respectively.

Let $\left(u_{l}, \rho_{l}\right)$ be left- and $(u, \rho)$ be right-hand states of either classical elementary waves or delta shock waves. Let us fix $\left(u_{l}, \rho_{l}\right)$ and compute the state $(u, \rho)$ that is connected on the right by either classical elementary waves or delta shock waves as given here (see figure 1).

The curves associated with 2-rarefaction waves are

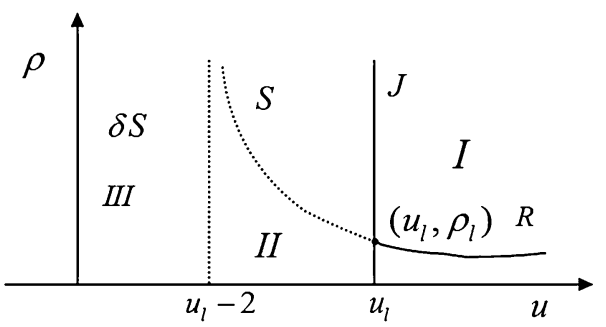

Figure 1. Riemann solution of (1) and (3) in $(u, \rho)$ plane.

$$
R\left(u_{l}, \rho_{l}\right)=\left\{\begin{array}{l}
\frac{d x}{d t}=\lambda_{2}=u \\
\rho=\rho_{l} e^{\left(u-u_{l}\right)} \\
u_{l}<u
\end{array}\right.
$$

and the curves associated with 2 -shock waves are

$$
S\left(u_{l}, \rho_{l}\right)=\left\{\begin{array}{c}
C=\frac{1}{2}\left(u+u_{l}\right), \\
\rho=\frac{2-u_{l}+u}{2+u_{l}-u} \rho_{l}, \\
u<u_{l}<u+2,
\end{array}\right.
$$

where $C$ represents the shock speed.

The desirable states that connect $\left(u_{l}, \rho_{l}\right)$ by a contact discontinuity on the right are given as follows:

$$
J\left(u_{l}, \rho_{l}\right)=\left\{\begin{array}{l}
\tau=u-1=u_{l}-1, \\
u=u_{l}
\end{array}\right.
$$

where $\tau$ represents the contact discontinuity speed.

It is not possible to obtain classical Riemann solution when $u_{l} \geq u_{r}+2$. In this case, the Riemann solution contains delta measure supported on a line. For measure solution, we have the following definition.

Definition 2.1 The two-dimensional weighted $\delta$-measure $f(r) \delta_{\Gamma}$ that has support on a smooth curve $\Gamma=$ $\{(x(r), t(r)): c<r<d\}$ can be defined by

$$
\left\langle f(r) \delta_{\Gamma}, \phi(x, t)\right\rangle=\int_{a}^{b} f(r) \phi(x(r), t(r)) d r
$$

for every test function $\phi \in C_{0}^{\infty}\left(\mathbb{R} \times \mathbb{R}_{+}\right)$.

Now, let us use the definition of delta shock wave solution introduced by Danilov and Shelkovich [12, 13] and given here.

Suppose that $\Gamma=\left\{\gamma_{i} \mid i \in I\right\}$ is a graph in the closed upper half-plane $\{(x, t) \mid x \in \mathbb{R}, t \geq 0\}$ that contains smooth $\operatorname{arcs} \gamma_{i}$, where $i \in I$ and $I$ is the finite index set. Let $I_{0}$ be a subset of $I$ containing all indices of arcs that connect to the $x$-axis and $\Gamma_{0}=\left\{x_{j}^{0}: j \in I_{0}\right\}$ be the set of initial points of the $\operatorname{arcs} \gamma_{j}$ with $j \in I_{0}$.

Definition 2.2 A pair of distributions $(u, \rho)$ and a graph $\Gamma$, where $\rho$ is of the form 


$$
\rho(x, t)=\hat{\rho}(x, t)+f(x, t) \delta(\Gamma),
$$

in which $u, \hat{\rho} \in L^{\infty}\left(\mathbb{R} \times \mathbb{R}_{+}\right)$and the singular part is given by

$$
f(x, t) \delta(\Gamma)=\sum_{i \in I} f_{i}(x, t) \delta\left(\gamma_{i}\right) .
$$

Consider the initial data of the form

$$
\begin{aligned}
(u, \rho)(x, 0)= & \left(u_{0}(x), \hat{\rho}_{0}(x)\right. \\
& \left.+\sum_{j \in I_{0}} f_{j}\left(x_{j}^{0}, 0\right) \delta\left(x-x_{j}^{0}\right)\right),
\end{aligned}
$$

in which $u_{0}, \hat{\rho}_{0} \in L^{\infty}(\mathbb{R})$; then $(u, \rho)$ is called delta shock wave solution for (1) with initial data (4) if

$$
\begin{aligned}
\int_{0}^{\infty} & \int_{-\infty}^{\infty}\left(u \phi_{t}+\frac{u^{2}}{2} \phi_{x}\right) d x d t \\
& +\int_{-\infty}^{\infty} u_{0}(x) \phi(x, 0) d x=0
\end{aligned}
$$

and

$$
\begin{aligned}
\int_{0}^{\infty} & \int_{-\infty}^{\infty}\left(\hat{\rho} \phi_{t}+(u-1) \hat{\rho} \phi_{x}\right) d x d t \\
& +\sum_{i \in I} \int_{\gamma_{i}} f_{i}(x, t) \frac{\partial \phi(x, t)}{\partial l} \\
& +\int_{-\infty}^{\infty} \hat{\rho}_{0}(x) \phi(x, 0) d x \\
& +\sum_{k \in I_{0}} f_{k}\left(x_{k}^{0}, 0\right) \phi\left(x_{k}^{0}, 0\right)=0
\end{aligned}
$$

hold for every test function $\phi \in C_{0}^{\infty}\left(\mathbb{R} \times \mathbb{R}_{+}\right)$, where $\frac{\partial \phi(x, t)}{\partial l}$ is tangential derivative of $\phi$ on the graph $\gamma_{i}$ and $\int_{\gamma_{i}} \cdot d l$ represents line integral over the arc $\gamma_{i}$.

From this definition, a delta shock wave solution of the Riemann problem (1) and (3) can be formed for the case $u_{l} \geq u_{r}+2$ in the following way:

$$
\begin{aligned}
u(x, t)= & \left\{\begin{array}{c}
u_{l}, x<\sigma t, \\
u_{r}, x>\sigma t,
\end{array}\right. \\
\rho(x, t)= & \left\{\begin{array}{c}
\rho_{l}, x<\sigma t, \\
\rho_{r}, x>\sigma t,
\end{array}\right\} \\
& +f(t) \delta(x-\sigma t),
\end{aligned}
$$

where

$$
\sigma=\frac{\left(u_{l}+u_{r}\right)}{2}, f(t)=\frac{1}{2}\left(\left(\rho_{r}+\rho_{l}\right)\left(u_{l}-u_{r}\right)+2\left(\rho_{r}-\rho_{l}\right)\right) t,
$$

are propagation speed and strength of delta shock wave, respectively.

The generalized Rankine-Hugoniot conditions

$$
\left\{\begin{array}{l}
\frac{d x(t)}{d t}=\sigma \\
\frac{d f(t)}{d t}=(\sigma[\rho]-[(u-1) \rho]), \\
\sigma[u]=\left[\frac{1}{2} u^{2}\right],
\end{array}\right.
$$

are satisfied by the delta shock wave solution (5) and (6), where $[\rho]=\rho_{r}-\rho_{l}$ denotes jump in $\rho$. The generalized Rankine-Hugoniot conditions show the relationship between the propagation speed, weight, location and the assignment of $u$ on its discontinuity. For uniqueness, the delta entropy conditions $\lambda_{1}\left(u_{r}\right) \leqslant \sigma \leqslant \lambda_{1}\left(u_{l}\right)$ and $\lambda_{2}\left(u_{r}\right) \leqslant$ $\sigma \leqslant \lambda_{2}\left(u_{l}\right)$ are imposed, which means that, in $(x, t)$ plane, all the characteristics lines on either side of delta shock wave run into the line of delta shock wave, which implies that delta shock wave is an overcompressive shock.

For the delta shock wave solution (5) with (6) constructed earlier, denoted by $\delta S$, the following must hold:

$$
\begin{aligned}
& \left\langle u, \phi_{t}\right\rangle+\left\langle\frac{u^{2}}{2}, \phi_{x}\right\rangle=0, \\
& \left\langle\rho, \phi_{t}\right\rangle+\left\langle(u-1) \rho, \phi_{x}\right\rangle=0,
\end{aligned}
$$

for every $\phi \in C_{0}^{\infty}\left(\mathbb{R} \times \mathbb{R}_{+}\right)$. From (7), as in [7, 23, 24], we have

$$
\begin{aligned}
\langle\rho, \phi\rangle= & \int_{0}^{\infty} \int_{-\infty}^{\infty} \rho_{*} \phi d x d t \\
& +\left\langle f(t) \delta_{S}, \phi\right\rangle, \\
\langle(u-1) \rho, \phi\rangle= & \int_{0}^{\infty} \int_{-\infty}^{\infty}\left(u_{*}-1\right) \rho_{*} \phi d x d t \\
& +\left\langle\left(u_{\delta}-1\right) f(t) \delta_{S}, \phi\right\rangle,
\end{aligned}
$$

in which $u_{*}=u_{l}+[u] H(x-\sigma t), \rho_{*}=\rho_{l}+[\rho] H(x-\sigma t)$ and $\left(u_{*}-1\right) \rho_{*}=\left(u_{l}-1\right) \rho_{l}+[(u-1) \rho] H(x-\sigma t)$. In the formula (8), $u_{\delta}=\frac{\left(u_{l}+u_{r}+2\right)}{2}=\sigma+1$ represents the value of $u$ on this delta shock wave curve $x=\sigma t$.

Hence, we can write the solution (5) as

$$
(u, \rho)(x, t)= \begin{cases}\left(u_{l}, \rho_{l}\right), & x<\sigma t \\ \left(\frac{\left(u_{l}+u_{r}+2\right)}{2}, v_{1}\right), & x=\sigma t \\ \left(u_{r}, \rho_{r}\right), & x>\sigma t\end{cases}
$$

where $\quad \sigma=\frac{\left(u_{l}+u_{r}\right)}{2} \quad$ and $\quad v_{1}=\left(\frac{\left(\rho_{r}+\rho_{l}\right)\left(u_{l}-u_{r}\right)+2\left(\rho_{r}-\rho_{l}\right)}{2}\right)$ $t \delta(x-\sigma t)$.

Depending upon the initial data, there exist three different wave patterns for Riemann solution of (1) and (3), which are given as

$$
\begin{aligned}
& J+R\left(u_{l}<u_{r}\right), J+S \\
& \quad\left(u_{r}<u_{l}<u_{r}+2\right), \delta S\left(u_{l} \geq u_{r}+2\right) .
\end{aligned}
$$




\section{Perturbed Riemann problem}

Now we consider the Riemann problem (2) and (3). The quasilinear form of (2) is

$$
V_{t}+A(V) V_{x}=0
$$

where

$$
\begin{aligned}
V & =\left[\begin{array}{c}
u \\
\rho
\end{array}\right], \\
A(V) & =\left[\begin{array}{cc}
u+\eta \alpha & 0 \\
\rho+\eta \beta & (u-1)+\eta \gamma
\end{array}\right] .
\end{aligned}
$$

The eigenvalues of (9) are $\lambda_{1}=u-1+\eta \gamma$ and $\lambda_{2}=u+\eta \alpha$ and the corresponding right eigenvectors are $\overrightarrow{r_{1}}=(0,1)^{t}$ and $\overrightarrow{r_{2}}=(\eta(\alpha-\gamma)+1, \rho+\eta \beta)^{t}$, respectively. Since $\nabla \lambda_{1}$. $\overrightarrow{r_{1}}=0$ and $\nabla \lambda_{2} \cdot \overrightarrow{r_{2}}=\{\eta(\alpha-\gamma)+1\} \neq 0$, the first and second characteristics fields are linearly degenerate and genuinely nonlinear, respectively; $z_{1}=u$ and $z_{2}=(\rho+$ $\eta \beta) \exp \left\{\frac{-u}{\eta(\alpha-\gamma)+1}\right\}$ are Riemann invariants, respectively.

Since the system (2) and Riemann data (3) are invariant under the transformation of coordinates

$$
(x, t) \rightarrow(m x, m t)(m \text { is a nonzero constant })
$$

we look for a self-similar solution of the form

$$
(u, \rho)(x, t)=(u, \rho)(\xi), \xi=\frac{x}{t} .
$$

The Riemann problem (2) and (3) becomes BVP for system of ODEs

$$
\left\{\begin{array}{l}
-\xi u_{\xi}+\left(\frac{u^{2}}{2}+\eta \alpha u\right)_{\xi}=0, \\
-\xi \rho_{\xi}+((u-1) \rho+\eta \beta u+\eta \gamma \rho)_{\xi}=0
\end{array}\right.
$$

with boundary conditions $(u, \rho)( \pm \infty)=\left(u_{r, l}, \rho_{r, l}\right)$.

Let $V=(u, \rho)^{t}$; then the system (10) reduces to the form

$$
A(V) V_{\xi}=0,
$$

where

$$
A(u, \rho)=\left[\begin{array}{cc}
-\xi+u+\eta \alpha & 0 \\
\rho+\eta \beta & -\xi+u-1+\eta \gamma
\end{array}\right],
$$

which implies that either $V=$ constant or $V$ is continuous solution of (11). Let us fix $\left(u_{l}, \rho_{l}\right)$ in the domain of hyperbolicity and compute the state $(u, \rho)$ that is connected on the right by either classical elementary waves or delta shock waves as given subsequently.

The curves associated with 2-rarefaction waves are

$$
\begin{aligned}
& R\left(u_{l}, \rho_{l}\right)= \\
& \left\{\begin{aligned}
& \xi=\lambda_{2}(u, \rho)=u+\eta \alpha, \\
&(\rho+\eta \beta) \exp \left\{\frac{-u}{1+\eta(\alpha-\gamma)}\right\}=\left(\rho_{l}+\eta \beta\right) \exp \left\{\frac{-u_{l}}{1+\eta(\alpha-\gamma)}\right\}, \\
& u>u_{l} .
\end{aligned}\right.
\end{aligned}
$$

The Rankine-Hugoniot jump conditions for bounded discontinuity at $\xi=C$ have the form

$$
\begin{aligned}
& C[u]=\left[\frac{u^{2}}{2}+\eta \alpha u\right], \\
& C[\rho]=[(u-1) \rho+\eta \beta u+\eta \gamma \rho],
\end{aligned}
$$

where $C=\frac{d x}{d t}$ and $[u]=u_{r}-u_{l}$.

From the first equation of (12), we have

$$
\left(C-\frac{u_{l}}{2}-\frac{u_{r}}{2}-\eta \alpha\right)\left(u_{r}-u_{l}\right)=0 .
$$

If $u_{l}=u_{r}$, then the second equation of (12) yields

$$
C=u_{l}-1+\eta \gamma=u_{r}-1+\eta \gamma,
$$

which corresponds to the contact discontinuity.

The desirable states that connect $\left(u_{l}, \rho_{l}\right)$ by a contact discontinuity on the right are given as follows:

$$
J\left(u_{l}, \rho_{l}\right)=\left\{\begin{array}{l}
\tau=u-1+\eta \gamma=u_{l}-1+\eta \gamma, \\
u=u_{l} .
\end{array}\right.
$$

The curves associated with 2-shock waves are

$$
S\left(u_{l}, \rho_{l}\right)=\left\{\begin{array}{l}
C=\frac{1}{2}\left(u+u_{l}\right)+\eta \alpha, \\
\rho=\frac{\rho_{l}\left(2+u-u_{l}+2 \eta(\alpha-\gamma)\right)+2 \eta \beta\left(u_{l}-u\right)}{\left(2+u_{l}-u+2 \eta(\alpha-\gamma)\right)} \\
u<u_{l}<u+2
\end{array}\right.
$$

When $u_{l} \geq u_{r}+2$, then a nonclassical situation appears, where Riemann problem (2) and (3) cannot be solved by classical waves. In this case, we observe that the singularity cannot be a jump with finite amplitude, i.e., there is no solution that is piecewise smooth and bounded. Hence, the Riemann solution of (2) and (3) contains a weighted $\delta$ measure supported on a line.

Depending upon initial data, the possible Riemann solution of (2) and (3) is given here.

Case i: If $u_{l}<u_{r}$, the Riemann solution contains a contact discontinuity $J$ and a rarefaction wave $R$ (see figure 2):

$$
(u, \rho)(x, t)=\left\{\begin{array}{l}
\left(u_{l}, \rho_{l}\right), \quad x<\left(u_{l}-1+\eta \gamma\right) t \\
\left(u_{*}, \rho_{*}\right) \quad\left(u_{l}-1+\eta \gamma\right) t<x<\left(u_{l}+\eta \alpha\right) t \\
R, \quad\left(u_{l}+\eta \alpha\right) t<x<\left(u_{r}+\eta \alpha\right) t \\
\left(u_{r}, \rho_{r}\right), \quad x>\left(u_{r}+\eta \alpha\right) t
\end{array}\right.
$$

where the state $(u, \rho)$ in $R$ is given by

$$
\begin{aligned}
(u, \rho)= & \left(\frac{x-\eta \alpha t}{t},\left(\rho_{r}+\eta \beta\right)\right. \\
& \left.\exp \left\{\frac{\left(x-\eta \alpha t-u_{r} t\right)}{(1+\eta(\alpha-\gamma)) t}\right\}-\eta \beta\right)
\end{aligned}
$$




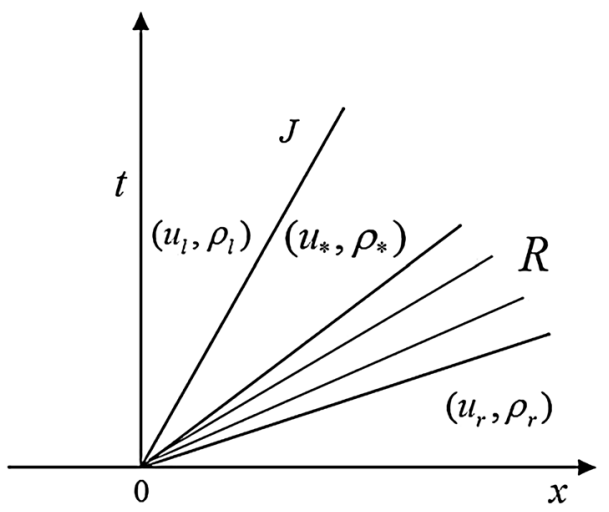

Figure 2. $J+R$ when $u_{l}<u_{r}$.

and intermediate state between $\mathrm{J}$ and $\mathrm{R}$ is

$$
\begin{aligned}
\left(u_{*}, \rho_{*}\right)= & \left(u_{l},\left(\rho_{r}+\eta \beta\right)\right. \\
& \left.\exp \left\{\frac{\left(u_{l}-u_{r}\right)}{1+\eta(\alpha-\gamma)}\right\}-\eta \beta\right) .
\end{aligned}
$$

Case ii: If $u_{r}<u_{l}<u_{r}+2$, the Riemann solution contains contact discontinuity $J$ and shock wave $S$ (see figure 3 )

$$
\begin{aligned}
& (u, \rho)(x, t) \\
& = \begin{cases}\left(u_{l}, \rho_{l}\right), & x<\left(u_{l}-1+\eta \gamma\right) t, \\
\left(u_{*}, \rho_{*}\right), & \left(u_{l}-1+\eta \gamma\right) t<x<\left(\frac{u_{l}+u_{r}+2 \eta \alpha}{2}\right) t, \\
\left(u_{r}, \rho_{r}\right), & x>\left(\frac{u_{l}+u_{r}+2 \eta \alpha}{2}\right) t,\end{cases}
\end{aligned}
$$

where $\left(u_{*}, \rho_{*}\right)=\left(u_{l}, \frac{\rho_{r}\left(2+u_{l}-u_{r}+2 \eta(\alpha-\gamma)\right)}{\left(2+u_{r}-u_{l}+2 \eta(\alpha-\gamma)+2 \eta \beta\left(u_{l}-u_{r}\right)\right)}\right)$ is an intermediate state between $J$ and $S$.

Case iii: When $u_{l} \geq u_{r}+2$, Riemann solution of (2) and (3) consists of delta shock wave that is as follows (see figure 4):

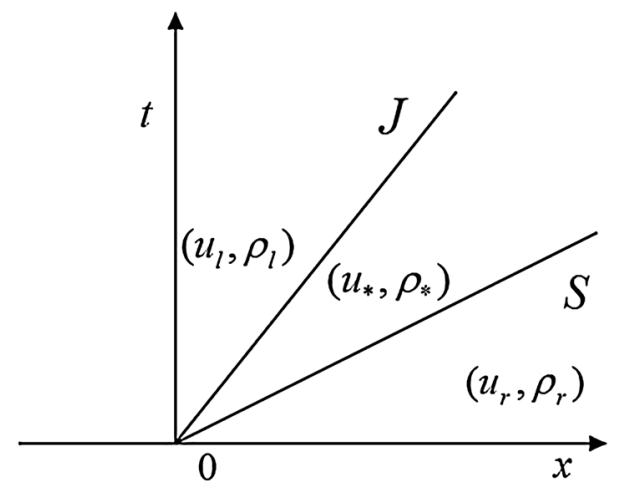

Figure 3. $J+S$ when $u_{r}<u_{l}<u_{r}+2$.

$$
(u, \rho)(x, t)= \begin{cases}\left(u_{l}, \rho_{l}\right), & x<\sigma t, \\ \left(u_{\delta}, f(t) \delta(x-\sigma t)\right), & x=\sigma t, \\ \left(u_{r}, \rho_{r}\right), & x>\sigma t,\end{cases}
$$

where $f(t)$ is the strength and $\sigma$ denotes speed of propagation of delta shock wave; $u_{\delta}$ specifies the value of $u$ on the delta shock wave curve. Actually, the delta shock wave solution (15) to the Riemann problem (2) and (3) must hold:

$$
\begin{aligned}
& \left\langle u, \phi_{t}\right\rangle+\left\langle\frac{u^{2}}{2}+\eta \alpha u, \phi_{x}\right\rangle=0, \\
& \left\langle\rho, \phi_{t}\right\rangle+\left\langle(u-1) \rho+\eta \beta u+\eta \gamma \rho, \phi_{x}\right\rangle=0,
\end{aligned}
$$

for every $\phi \in C_{0}^{\infty}\left(\mathbb{R} \times \mathbb{R}_{+}\right)$. For delta shock waves, we propose the following theorem.

Theorem 3.1 If $u_{l} \geq u_{r}+2$, then the Riemann solution of (2) and (3) consists of delta shock wave, which is written in the form (15) where

$$
\begin{aligned}
u_{\delta}= & \frac{1}{2}\left(u_{l}+u_{r}+2+2 \eta(\alpha-\gamma)\right), \\
\sigma= & \frac{1}{2}\left(u_{l}+u_{r}+2 \eta \alpha\right), \\
f(t)= & \frac{1}{2}\left(\left(\rho_{r}+\rho_{l}+2 \eta \beta\right)\left(u_{l}-u_{r}\right)\right. \\
& \left.+(2+2 \eta(\alpha-\gamma))\left(\rho_{r}-\rho_{l}\right)\right) t .
\end{aligned}
$$

Furthermore, the generalized Rankine-Hugoniot jump conditions

$$
\left\{\begin{array}{l}
\frac{d x(t)}{d t}=\sigma \\
\frac{d f(t)}{d t}=(\sigma[\rho]-[(u-1) \rho+\eta \beta u+\eta \gamma \rho]), \\
\sigma[u]=\left[\frac{u^{2}}{2}+\eta \alpha u\right]
\end{array}\right.
$$

must be satisfied by the delta shock wave solution (15) and (16). For uniqueness, the delta entropy conditions $\lambda_{1}\left(u_{r}\right) \leqslant$ $\sigma \leqslant \lambda_{1}\left(u_{l}\right)$ and $\lambda_{2}\left(u_{r}\right) \leqslant \sigma \leqslant \lambda_{2}\left(u_{l}\right)$ are imposed.

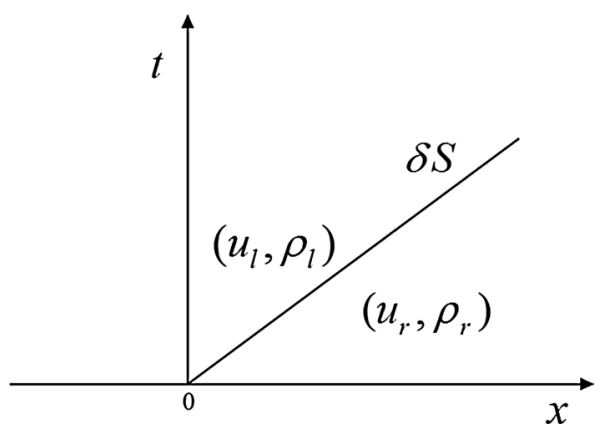

Figure 4. $\delta S$ when $u_{l} \geq u_{r}+2$. 
Proof In order to prove that (15) is a solution of (2) and (3) in the sense of distributions, it is enough to prove the following integral identities:

$$
\begin{gathered}
\int_{0}^{\infty} \int_{-\infty}^{\infty}\left(u \phi_{t}+\left(\frac{u^{2}}{2}+\eta \alpha u\right) \phi_{x}\right) d x d t=0, \\
\int_{0}^{\infty} \int_{-\infty}^{\infty}\left(\rho \phi_{t}+((u-1) \rho+\eta \beta u\right. \\
\left.+\eta \gamma \rho) \phi_{x}\right) d x d t=0
\end{gathered}
$$

hold for every $\phi \in C_{0}^{\infty}\left(\mathbb{R} \times \mathbb{R}_{+}\right)$.

Without loss of generality, let us choose $\sigma>0$; then we have

$$
\begin{aligned}
I= & \int_{0}^{\infty} \int_{-\infty}^{\infty}\left(u \phi_{t}+\left(\frac{u^{2}}{2}+\eta \alpha u\right) \phi_{x}\right) d x d t \\
= & \int_{0}^{\infty} \int_{-\infty}^{\sigma t}\left(u_{l} \phi_{t}+\left(\frac{u_{l}^{2}}{2}+\eta \alpha u_{l}\right) \phi_{x}\right) d x d t \\
& +\int_{0}^{\infty} \int_{\sigma t}^{\infty}\left(u_{r} \phi_{t}+\left(\frac{u_{r}^{2}}{2}+\eta \alpha u_{r}\right) \phi_{x}\right) d x d t \\
= & \int_{0}^{\infty} \int_{\frac{x}{\sigma}}^{\infty} u_{l} \phi_{t} d t d x+\int_{0}^{\infty} \int_{0}^{\frac{x}{\sigma}} u_{r} \phi_{t} d t d x \\
& +\int_{0}^{\infty}\left(\frac{u_{l}^{2}}{2}+\eta u_{l}-\frac{u_{r}^{2}}{2}-\eta \alpha u_{r}\right) \phi(\sigma t, t) d t \\
= & \int_{0}^{\infty}\left(\sigma\left(u_{r}-u_{l}\right)-\left(\left(\frac{u_{r}^{2}}{2}-\frac{u_{l}^{2}}{2}\right)\right.\right. \\
& \left.\left.+\eta \alpha\left(u_{r}-u_{l}\right)\right)\right) \phi(\sigma t, t) d t \\
= & 0 .
\end{aligned}
$$

Similarly

$$
\begin{aligned}
I I= & \int_{0}^{\infty} \int_{-\infty}^{\infty}\left(\rho \phi_{t}+((u-1) \rho\right. \\
& \left.+\eta \beta u+\eta \gamma \rho) \phi_{x}\right) d x d t \\
= & \int_{0}^{\infty} \int_{-\infty}^{\sigma t}\left(\rho_{l} \phi_{t}+\left(\left(u_{l}-1\right) \rho_{l}+\eta \beta u_{l}\right.\right. \\
& \left.\left.+\eta \gamma \rho_{l}\right) \phi_{x}\right) d x d t \\
& +\int_{0}^{\infty} \int_{\sigma t}^{\infty}\left(\rho_{r} \phi_{t}\right. \\
& \left.+\left(\left(u_{r}-1\right) \rho_{r}+\eta \beta u_{r}+\eta \gamma \rho_{r}\right) \phi_{x}\right) d x d t \\
& +\int_{0}^{\infty}\left(f ( t ) \left(\phi_{t}(\sigma t, t)\right.\right. \\
& \left.+\left(u_{\delta}-1+\eta \gamma\right) \phi_{x}(\sigma t, t)\right) d t \\
& +\int_{0}^{\infty} \eta \beta u_{\delta} \phi_{x}(\sigma t, t) d t \\
= & \int_{0}^{\infty} \int_{-\infty}^{0}\left(\rho_{l} \phi_{t}+\left(\left(u_{l}-1\right) \rho_{l}+\eta \beta u_{l}\right.\right. \\
& \left.\left.+\eta \gamma \rho_{l}\right) \phi_{x}\right) d x d t
\end{aligned}
$$

$+\int_{0}^{\infty} \int_{0}^{\sigma t}\left(\rho_{l} \phi_{t}+\left(\left(u_{l}-1\right) \rho_{l}\right.\right.$

$\left.\left.+\eta \beta u_{l}+\eta \gamma \rho_{l}\right) \phi_{x}\right) d x d t$

$+\int_{0}^{\infty} \int_{\sigma t}^{\infty}\left(\rho_{r} \phi_{t}+\left(\left(u_{r}-1\right) \rho_{r}\right.\right.$

$\left.\left.+\eta \beta u_{r}+\eta \gamma \rho_{r}\right) \phi_{x}\right) d x d t$

$+\int_{0}^{\infty}\left(f(t)\left(\phi_{t}(\sigma t, t)+\left(u_{\delta}-1\right.\right.\right.$

$\left.+\eta \gamma) \phi_{x}(\sigma t, t)\right) d t$

$=\int_{0}^{\infty} \int_{0}^{\sigma t} \rho_{l} \phi_{t} d x d t$

$+\int_{0}^{\infty} \int_{-\infty}^{\sigma t}\left(\left(u_{l}-1\right) \rho_{l}\right.$

$\left.+\eta \beta u_{l}+\eta \gamma \rho_{l}\right) \phi_{x} d x d t$

$+\int_{0}^{\infty} \int_{\sigma t}^{\infty} \rho_{r} \phi_{t} d x d t$

$+\int_{0}^{\infty} \int_{\sigma t}^{\infty}\left(\left(u_{r}-1\right) \rho_{r}\right.$

$\left.+\eta \beta u_{r}+\eta \gamma \rho_{r}\right) \phi_{x} d x d t$

$+\int_{0}^{\infty}\left(f(t)\left(\phi_{t}(\sigma t, t)+\left(u_{\delta}-1\right.\right.\right.$

$\left.+\eta \gamma) \phi_{x}(\sigma t, t)\right) d t$

$=-\int_{0}^{\infty} \rho_{l} \phi\left(x, \frac{x}{\sigma}\right) d x$

$+\int_{0}^{\infty} \rho_{r} \phi\left(x, \frac{x}{\sigma}\right) d x$

$-\int_{0}^{\infty}\left(\left(\left(u_{r}-1\right) \rho_{r}\right.\right.$

- $\left.\left.\left(u_{l}-1\right) \rho_{l}\right)\right) \phi(\sigma t, t) d t$

$-\int_{0}^{\infty}\left(\eta \beta\left(u_{r}-u_{l}\right)\right.$

$\left.+\eta \gamma\left(\rho_{r}-\rho_{l}\right)\right) \phi(\sigma t, t) d t$

$$
\begin{aligned}
+ & \int_{0}^{\infty} f(t) d \phi(\sigma t, t) \\
= & \int_{0}^{\infty}\left(\sigma\left(\rho_{r}-\rho_{l}\right)\right. \\
& -\left(\left(u_{r}-1\right) \rho_{r}-\left(u_{l}-1\right) \rho_{l}\right) \phi(\sigma t, t) d t \\
& -\int_{0}^{\infty}\left(\eta \beta\left(u_{r}-u_{l}\right)\right. \\
& \left.\left.+\eta \gamma\left(\rho_{r}-\rho_{l}\right)\right)+f^{\prime}(t)\right) \phi(\sigma t, t) d t \\
= & 0
\end{aligned}
$$

holds for every $\phi \in C_{0}^{\infty}\left(\mathbb{R} \times \mathbb{R}_{+}\right)$, where $\sigma=u_{\delta}-1$ $+\eta \gamma$. 


\section{Limit of Riemann solution of (2) and (3) when $\eta \rightarrow 0$}

Here, we prove that limit of the solution of Riemann problem (2) and (3) converges to solution of (1) and (3), when the perturbation parameter $\eta \rightarrow 0$.

Theorem 4.1 Let $\left(u_{l, r}, \rho_{l, r}\right)$ be given Riemann initial data. The limit of the Riemann solution of (2) is exactly the same as the solution of (1).

Proof We divide the proof into three cases depending upon the initial data.

Case I: If $u_{l}<u_{r}$, the Riemann solution of (2) and (3) consists of a contact discontinuity and a rarefaction wave. From (13), we take limit $\eta$ tends to zero; we have

$\lim _{\eta \rightarrow 0}(u, \rho)(x, t)= \begin{cases}\left(u_{l}, \rho_{l}\right), & x<\left(u_{l}-1\right) t, \\ \left(u_{l}, \rho_{r} e^{\left(u_{l}-u_{r}\right)}\right), & \left(u_{l}-1\right) t \leq x \leq u_{l} t \\ \left(\frac{x}{t}, \rho_{r} e^{\left(\frac{x}{t}-u_{r}\right)}\right), & u_{l} t<x<u_{r} t, \\ \left(u_{r}, \rho_{r}\right), & x>u_{r} t,\end{cases}$

which is the same as the Riemann solution of (1) and (3) when $u_{l}<u_{r}$.

Case II: If $u_{r}<u_{l}<u_{r}+2$, from (14), the Riemann solution of (2) and (3) is $J+S$. As $\eta$ tends to zero, we obtain

$$
\begin{aligned}
& \lim _{\eta \rightarrow 0}(u, \rho)(x, t) \\
& = \begin{cases}\left(u_{l}, \rho_{l}\right), & x<\left(u_{l}-1\right) t, \\
\left(u_{l}, \frac{\rho_{r}\left(2+u_{l}-u_{r}\right)}{\left(2+u_{r}-u_{l}\right)}\right), & \left(u_{l}-1\right) t<x<\frac{1}{2}\left(u_{l}+u_{r}\right) t, \\
\left(u_{r}, \rho_{r}\right), & x>\frac{1}{2}\left(u_{l}+u_{r}\right) t,\end{cases}
\end{aligned}
$$

which is the same as the Riemann solution of (1) and (3) when $u_{r}<u_{l}<u_{r}+2$.

Case III: If $u_{l} \geq u_{r}+2$, the Riemann solution of (2) and (3) is delta shock wave. From (15) and (16), we take limit $\eta$ tends to zero; we obtain

$$
\lim _{\eta \rightarrow 0}(u, \rho)(x, t)=\left\{\begin{array}{cc}
\left(u_{l}, \rho_{l}\right), & x<\left(\frac{u_{l}+u_{r}}{2}\right) t \\
\left(u_{1}, \rho_{1}\right), & x=\left(\frac{u_{l}+u_{r}}{2}\right) t \\
\left(u_{r}, \rho_{r}\right), & x>\left(\frac{u_{l}+u_{r}}{2}\right) t
\end{array}\right.
$$

where $\quad\left(u_{1}, \rho_{1}\right)=\left(\frac{u_{l}+u_{r}+2}{2},\left(\frac{\left(\rho_{r}+\rho_{l}\right)\left(u_{l}-u_{r}\right)+2\left(\rho_{l}-\rho_{r}\right)}{2}\right) t \delta(x-\right.$ $\sigma t)$ ), which is the same as the Riemann solution of (1) and (3) when $u_{l} \geq u_{r}+2$.

\section{Conclusions}

It is noticed that the limit of Riemann solution of (2)-(3) converges to the corresponding solution of the Riemann problem (1) and (3) as $\eta \rightarrow 0$. We prove that the perturbation in flux functions does not change the structure of Riemann solution. We conclude that the Riemann solution (1) and (3) is stable under triangular linear approximation of flux functions.

\section{Acknowledgements}

We thank the referees for their valuable comments and suggestions. The first author (Anupam Sen) is supported by University Grant Commission, Government of India (Sr. No. 2121540947, Ref. No. 20/12/2015(ii)EU-V). The third author (Dia Zeidan) has been supported by the Scientific Research Support Fund, Ministry of Higher Education \& Scientific Research through the German Jordanian University (Fund No. Bas/1/05/2016), Amman, Jordan.

\section{References}

[1] Nedeljkov M and Oberguggenberger M 2008 Interactions of delta shock waves in a strictly hyperbolic system of conservation laws. J. Math. Anal. Appl. 344(2): 11431157

[2] Hayes B T and LeFloch P G 1996 Measure solutions to a strictly hyperbolic system of conservation laws. Nonlinearity 9(6): 1547-1563

[3] Keyfitz B L and Kranzer H C 1995 Spaces of weighted measures for conservation laws with singular shock solutions. J. Diff. Equ. 118(2): 420-451

[4] Sen A, Raja Sekhar T and Sharma V D 2017 Wave interactions and stability of the Riemann solution for a strictly hyperbolic system of conservation laws. Q. Appl. Math. 75(3): 539-554

[5] Lax P D 1957 Hyperbolic systems of conservation laws II. Commun. Pure Appl. Math. 10(4): 537-566

[6] Glimm J 1965 Solutions in the large for nonlinear hyperbolic systems of equations. Commun. Pure Appl. Math. 18(4): 697-715

[7] Tan D, Zhang T and Zheng Y 1994 Delta-shock waves as limits of vanishing viscosity for hyperbolic systems of conservation laws. J. Differ. Equ. 112(1): 1-32

[8] Joseph K T 1993 A Riemann problem whose viscosity solutions contain $\delta$-measures. Asymptotic Anal. 7(2): $105-120$

[9] Sen A and Raja Sekhar T 2019 Delta shock wave as selfsimilar viscosity limit for a strictly hyperbolic system of conservation laws. J. Math. Phys. 60(5): 051510, 12 pp

[10] Chen G Q and Liu H 2003 Formation of $\delta$-shocks and vacuum states in the vanishing pressure limit of solutions to the Euler equations for isentropic fluids. SIAM J. Math. Anal. 34(4): 925-938 
[11] Chen G Q and Liu H 2004 Concentration and cavitation in the vanishing pressure limit of solutions to the Euler equations for nonisentropic fluids. Physica D: Nonlin. Phenom. 189(1-2): 141-165

[12] Danilov V G and Shelkovich V M 2005 Dynamics of propagation and interaction of $\delta$-shock waves in conservation law systems. J. Differ. Equ. 211(2): 333-381

[13] Danilov V G and Shelkovich V M 2005 Delta-shock wave type solution of hyperbolic systems of conservation laws. $Q$. Appl. Math. 63(3): 401-427

[14] Kalisch H and Teyekpiti V 2018 A shallow-water system with vanishing buoyancy. Appl. Anal., https://doi.org/ $10.1080 / 00036811.2018 .1546000$

[15] Yin G and Sheng W 2009 Delta shocks and vacuum states in vanishing pressure limits of solutions to the relativistic Euler equations for polytropic gases. J. Math. Anal. Appl. 355(2): 594-605

[16] Mitrović D and Nedeljkov M 2007 Delta shock waves as a limit of shock waves. J. Hyperbolic Differ. Equ. 4(4): 629-653

[17] Sun M 2018 The limits of Riemann solutions to the simplified pressureless Euler system with flux approximation. Math. Methods Appl. Sci. 41(12): 4528-4548
[18] Shen C 2011 The limits of Riemann solutions to the isentropic magnetogasdynamics. Appl. Math. Lett. 24(7): $1124-1129$

[19] Sun M 2016 Structural stability of solutions to the Riemann problem for a non-strictly hyperbolic system with flux approximation. Electron. J. Differ. Equ. 2016(126): 1-16

[20] Shen C and Sun M 2019 The singular limits of Riemann solutions to a chemotaxis model with flux perturbation. $Z$. Angew. Math. Mech. 99: e201800046, 28 pp

[21] Smith T A, Petty D J and Pantano C 2016 A Roe-like numerical method for weakly hyperbolic systems of equations in conservation and non-conservation form. J. Comput. Phys. 316: 117-138

[22] Yang H and Liu J 2015 Delta-shocks and vacuums in zeropressure gas dynamics by the flux approximation. Sci. China Math. 58(11): 2329-2346

[23] Sen A and Raja Sekhar T 2019 Structural stability of the Riemann solution for a strictly hyperbolic system of conservation laws with flux approximation. Commun. Pure Appl. Anal. 18(2): 931-942

[24] Sheng W and Zhang T 1999 The Riemann problem for the transportation equations in gas dynamics. Mem. Am. Math. Soc. 654 